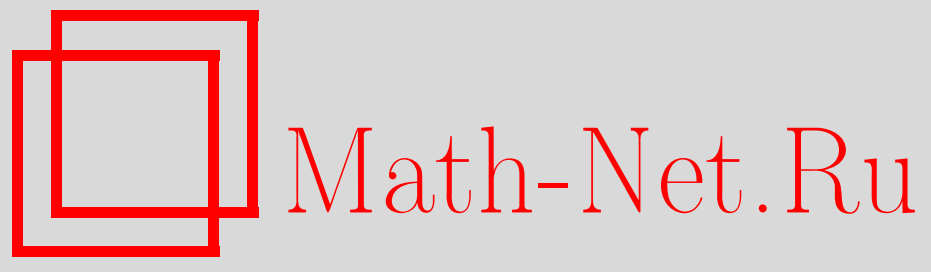

А. В. Колесников, Интегрируемость оптимальных отображений, Матем. заметки, 2006, том 80, выпуск 4, 546560

DOI: https://doi.org/10.4213/mzm2847

Использование Общероссийского математического портала Math-Net.Ru подразумевает, что вы прочитали и согласны с пользовательским соглашением http://www . mathnet.ru/rus/agreement

Параметры загрузки:

IP : 54.237 .206 .68

26 апреля 2023 г., 16:41:55

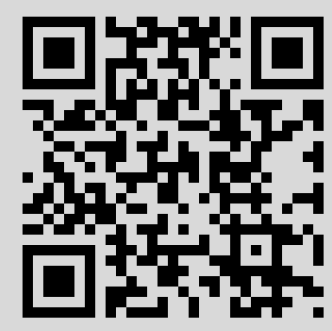




\section{ИНТЕГРИРУЕМОСТЬ ОПТИМАЛЬНЫХ ОТОБРАЖЕНИЙ}

\section{А. В. Колесников}

В работе изучается интегрируемость оптимального отображения $T$, отображающего вероятностную меру $\mu$ в меру $g \cdot \mu$. Мы предполагаем, что $T$ минимизирует функцию стоимости $c$ и $\mu$ удовлетворяет некоторым специальным неравенствам, связанным с $c$ (неравенству для инфимум-свертки или логарифмическому $c$-неравенству Соболева). Полученные результаты применяются для анализа мер вида $\exp \left(-|x|^{\alpha}\right)$.

Библиография: 25 названий.

1. Введение. В работе рассматривается вероятностная мера $\mu$ на $\mathbb{R}^{d}$ и оптимальное отображение $T: \mathbb{R}^{d} \rightarrow \mathbb{R}^{d}$,

$$
T(x)=x+F(x),
$$

отображающее $\mu$ в другую вероятностную меру $g \cdot \mu$. Последнее означает, что

$$
\int_{\mathbb{R}^{d}} \psi(T) d \mu=\int_{\mathbb{R}^{d}} \psi g d \mu
$$

для любой борелевской функции $\psi$. Оптимальность означает, что среди множества всех отображений $t: \mathbb{R}^{d} \rightarrow \mathbb{R}^{d}$, переводящих $\mu$ в $g \cdot \mu$, функционал

$$
t \rightarrow \int_{\mathbb{R}^{d}} c(t(x)-x) d \mu,
$$

достигает минимума на $T$. Здесь $c$ - некоторая неотрицательная функция, называемая функиией стоимости. Во многих ситуациях $T$ можно найти, решая известную вариационную задачу Монжа-Канторовича: в пространстве вероятностных мер на $\mathbb{R}^{d} \times \mathbb{R}^{d}$ с заданными проекциями $\mu$ и $g \cdot \mu$ на первый и второй множители соответственно требуется найти меру, минимизирующую следующий функционал:

$$
m \rightarrow \int_{\mathbb{R}^{d} \times \mathbb{R}^{d}} c(x-y) m(d x d y) .
$$

Если $m$ - искомая мера, то величина

$$
W_{c}(\mu, g \cdot \mu)=\int_{\mathbb{R}^{d} \times \mathbb{R}^{d}} c(x-y) m(d x d y)
$$

Работа выполнена при поддержке Российского фонда фундаментальных исследований, грант № 04-01-00748, и DFG, грант № 436 RUS 113/343/0(R). Автор признателен за поддержку Центру математических исследований Эннио Де Джорджи, Высшая Нормальная Школа, Пиза.

(C) А. В. Колесников, 2006 
называется стоимостъю транспортировки.

Известно, что для многих функций стоимости $c$ решение $m$ оказывается сосредоточенным на графике некоторого отображения: $m\left(\mathbb{R}^{2 d} \backslash\left\{(x, T(x)), x \in \mathbb{R}^{d}\right\}\right)=0$. Именно это отображение $T$ и является искомым оптимальным отображением.

Особенно хорошо изучены свойства оптимальных отображений для $c=|x|^{2}$. В этом случае согласно результатам [1], [2] (для более подробного знакомства см. книги [3]-[5]) при незначительных ограничениях на меры $\mu, g \cdot \mu$ (достаточно считать, что $\mu$ абсолютно непрерывна) $T$ существует и единственно с точностью до множества $\mu$-меры нуль. Кроме того, $T$ имеет вид $T=\nabla V$, где $V$ - выпуклая функция.

В дальнейшем мы предполагаем, что $\mu$ удовлетворяет некоторым специальным неравенствам, связанным с $c$; например, неравенству для инфимум-свертки или логарифмическому $c$-неравенству Соболева. Напомним, что мера $\mu$ на $\mathbb{R}^{d}$ удовлетворяет логарифмическому неравенству Соболева, если для гладкой функции $f$

$$
\operatorname{Ent}_{\mu} f^{2} \leqslant 2 C \int_{\mathbb{R}^{d}}|\nabla f|^{2} d \mu
$$

где

$$
\operatorname{Ent}_{\mu} g:=\int_{\mathbb{R}^{d}} g \log g d \mu-\left(\int_{\mathbb{R}^{d}} g d \mu\right) \log \int_{\mathbb{R}^{d}} g d \mu .
$$

Основным примером меры, удовлетворяющей этому неравенству с $C=1$, является стандартная гауссовская мера. Логарифмическое неравенство Соболева, впервые доказанное Гроссом для гауссовских мер в [6], имеет обширные приложения в анализе на винеровском пространстве (см. [7]). Много работ посвящены выяснению условий, при которых мера удовлетворяет (1.1). Хорошо известно следующее необходимое условие:

$$
\int_{\mathbb{R}^{d}} e^{\varepsilon|x|^{2}} d \mu<\infty
$$

для некоторого $\varepsilon>0$. Согласно результату [8] это условие является достаточным, если мера выпукла, т.е. имеет плотность $e^{-V} d x$, где $V$ - выпуклая функция. Подробнее о логарифмическом неравенстве Соболева и его приложениях в теории вероятностей и анализе см. [9], [10].

Применяя $(1.1)$ к $1+\varepsilon f$, получаем в пределе при $\varepsilon \rightarrow 0$ неравенство Пуанкаре

$$
\int_{\mathbb{R}^{d}} f^{2} d \mu-\left(\int_{\mathbb{R}^{d}} f d \mu\right)^{2} \leqslant C \int_{\mathbb{R}^{d}}|\nabla f|^{2} d \mu .
$$

Хорошо известно, что всякая мера, удовлетворяющая (1.1), удовлетворяет двум важным неравенствам. Это неравенство инфимум-свертки

$$
\int_{\mathbb{R}^{d}} \exp Q_{C} f d \mu \leqslant \exp \int_{\mathbb{R}^{d}} f d \mu, \quad Q_{C} f(x)=\inf _{y}\left[f(y)+\frac{|x-y|^{2}}{2 C}\right]
$$

и транспортное неравенство

$$
\int_{\mathbb{R}^{d}}|F|^{2} d \mu \leqslant 2 C \operatorname{Ent}_{\mu} g
$$

где $T=\mathrm{Id}+F$ - оптимальное отображение $\mu$ в $g \cdot \mu$, соответствующее функции стоимости $c=|x|^{2}$ (см. [11], [9]). Неравенство (1.5) было доказано Талаграном в [12] 
для стандартной гауссовской меры и обобщено в [13] на случай меры, удовлетворяющей (1.1).

Неравенства типа (1.5) приобрели большую популярность в связи с изучением концентрации мер (см. [9]). Известно, что меры, удовлетворяющие (1.5), обладают свойством гауссовской концентрации. Напомним также известное неравенство Пинскера-Кульбака-Чизара (см. [7]) для расстояния по вариации $\|\cdot\|_{T V}$ для произвольных вероятностных мер $\mu, \nu$ :

$$
\|\mu-\nu\|_{T V} \leqslant \sqrt{2 \operatorname{Ent}_{\mu}\left(\frac{d \nu}{d \mu}\right)} .
$$

Известно, что это неравенство является транспортным неравенством для дискретной метрики (см. [14]).

За последнее время появилось много обобщений транспортных неравенств. Для подробного знакомства см. работы [14]-[16] и библиографию в них. Среди прочих результатов были получены транспортные неравенства для мер, удовлетворяющих более слабым ограничениям, чем неравенства типа Соболева или изопериметрические неравенства (например, для мер, удовлетворяющих (1.2)). Общий вид таких неравенств

$$
W_{c}(\mu, \nu) \leqslant \alpha\left(\operatorname{Ent}_{\mu}\left(\frac{d \nu}{d \mu}\right)\right),
$$

где $\alpha$ - некоторая неотрицательная возрастающая функция.

В этой работе изучаются неравенства типа

$$
\int_{\mathbb{R}^{d}} P(T(x)-x) d \mu \leqslant \int_{\mathbb{R}^{d}} Q(g) d \mu,
$$

где $P, Q$ - некоторые неотрицательные функции. Заметим, что неравенство (1.5) является неравенством такого типа. Интересное наблюдение было сделано Ферником в [17], который рассмотрел гауссовскую меру $\gamma$ на сепарабельном пространстве Фреше и вероятностную меру $g \cdot \gamma$. При этом предполагалось, что $g \in L^{p}(\gamma)$ для некоторого $p>1$. В [17] было показано, что существует такое отображение $T=U+S$, где $U$ сохраняет меру $\gamma$ и $S$ - отображение со значениями в пространстве Камерона-Мартина $H$ меры $\gamma$, что функция $\exp \left(\omega|S|_{H}^{2}\right)$ интегрируема для достаточно малого $\omega$ (при этом $T$ не обязательно оптимально). Этот результат был обобщен в [18]. В частности, была доказана следуюшая теорема.

Теорема 1. Предположим, что н удовлетворяет логарифмическому неравенству Соболева (1.1). Рассмотрим оптимальное отображение $T(x)=x+F(x)$, отображающее $\mu$ в $g \cdot \mu$ и минимизирующее $c(x)=|x|^{2}$. Eсли $g \in L^{p}(\mu)$, где $p>1$, mo

$$
\int_{\mathbb{R}^{d}} \exp \left(\omega|F|^{2}\right) d \mu \leqslant\|g\|_{L^{p}(\mu)}
$$

для достаточно малого $\omega=\omega(C, p)$.

Аналогичным образом $\int_{\mathbb{R}^{d}}|F|^{2 p} d \gamma$ оценивается через $\int_{\mathbb{R}^{d}} g|\log g|^{p} d \gamma$, где $p \geqslant 1$. Кроме того, были получены некоторые точные оценки в гауссовском случае (для 
различных видов отображений, в том числе неоптимальных) и некоторые оценки для мер, удовлетворяющих неравенству Пуанкаре. Все оценки не зависят от размерности и в гауссовском случае верны также на бесконечномерных пространствах (с заменой стандартной нормы на норму пространства Камерона-Мартина).

В этой работе мы дадим обобщение теоремы 1 на случай неквадратичной функции стоимости. В качестве основного примера мы рассмотрим меру

$$
\mu_{\alpha}=\frac{1}{Z_{\alpha}^{d}} \prod_{i=1}^{d} e^{-\left|x_{i}\right|^{\alpha}} d x_{i}
$$

где $Z_{\alpha}=\int_{\mathbb{R}} e^{-|x|^{\alpha}} d x$ и $1<\alpha \leqslant 2$. В доказательстве используются недавние результаты работы [19] о логарифмическом неравенстве Соболева для мер вида (1.6).

2. Основные результаты. Для подробного знакомства с теорией Монжа-Канторовича для неквадратичных функций стоимости см. [3]-[5]. Ниже мы напомним основные результаты. Рассмотрим функцию стоимости $c(x)$ на $\mathbb{R}^{d}$. Всюду далее мы предполагаем:

A1) $c$ - неотрицательная, четная функция и $c(0)=0$;

A2) $c$ строго выпукла; это значит, что $c$ выпукла и равенство

$$
c(t x+(1-t) y)=t c(x)+(1-t) c(y)
$$

влечет $x=y$, или $t=0$, или $t=1$;

A3) с суперлинейна, т.е.

$$
\lim _{x \rightarrow \infty} \frac{c(x)}{|x|}=\infty
$$

A4) для любых $r>0$ и $\theta \in(0, \pi)$ и любой достаточно далекой от начала координат точки $p \in \mathbb{R}^{d}$ существует такое направление $z \in \mathbb{R}^{d}$, что на усеченном конусе

$$
K=\left\{x \in \mathbb{R}^{d},|x-p||z| \cos \left(\frac{\theta}{2}\right) \leqslant\langle z, x-p\rangle \leqslant r|z|\right\},
$$

$c$ достигает максимума в $p$.

Условия A2)-A4) были введены в [20], откуда мы используем результаты существования и регулярности.

Важным примером функции, удовлетворяющей условиям выше, является функция

$$
c(x)=\sum_{i=1}^{d}\left|x_{i}\right|^{p}
$$

где $1<p \leqslant 2$. Однако для изучения транспортных неравенств более удобна функция стоимости $\tilde{c}$, квадратичная при малых $x$ и совпадающая с $c$ при больших $x$ (см. пример ниже).

Для произвольной функции $c$ определим сопряженную функцию

$$
c^{*}(x)=\sup _{y \in \mathbb{R}^{d}}(\langle x, y\rangle-c(y)) .
$$

Пусть $\mu, g \cdot \mu$ - абсолютно непрерывные вероятностные меры, причем

$$
W_{c}(\mu, g \cdot \mu)=\inf _{m} \int_{\left(\mathbb{R}^{d}\right)^{2}} c(x-y) d m<\infty,
$$


и инфимум берется по всем мерам на $\left(\mathbb{R}^{d}\right)^{2}$ с проекциями $\mu$ и $g \cdot \mu$. Известно, что при условиях $\mathbf{A 2}$ )-A4) существует такое оптимальное отображение $T=\mathrm{Id}+F$, что T отображает $\mu$ в $g \cdot \mu$ и

$$
\int_{\mathbb{R}^{d}} c(F) d \mu=W_{c}(\mu, g \cdot \mu) .
$$

Известно (см. [20] или теорему 2.44 в [5]), что $T$ имеет вид

$$
T(x)=x+\nabla c^{*}(\nabla \Phi) \quad \mu \text {-п.в. }
$$

для некоторой $c$-выпуклой функции $\Phi$. Последнее означает, что

$$
-\Phi=Q_{c} \Psi
$$

для некоторой $\Psi$, где

$$
Q_{c} f(x)=\inf _{y}[f(y)+c(x-y)]
$$

- инфимум-свертка. Обозначим $\Phi^{c}=-Q_{c} \Phi$. В силу дуальности Лежандра $\Psi=$ $\Phi^{c}$. Согласно результату из [20] существует такое выпуклое множество $K$, что $\Phi$ локально липшицева на $\Omega=\operatorname{Int}(K)$ и

$$
\Omega \subseteq \operatorname{Dom}(\Phi) \subseteq K
$$

следовательно, (2.1) корректно определено. Заметим, что в отличие от стандартного пути, мы не используем представление $T(x)=x-\nabla c^{*}(\nabla \tilde{\Phi})$, где $\tilde{\Phi}-c$-вогнутая функция. Тем не менее, так как $c$ предполагается четной, наше представление (2.1) равносильно стандартному.

Мы будем использовать хорошо известные соотношения

$$
\begin{gathered}
\Phi(x)+\Phi^{c}(y) \geqslant-c(x-y), \\
\Phi+\Phi^{c}(T(x))=-c(T(x)-x) \quad \text { для } \mu \text {-п.в. } x .
\end{gathered}
$$

Будем говорить, что $\mu$ удовлетворяет неравенству инфимум-свертки, если для любой измеримой ограниченной функции $f$

$$
\int_{\mathbb{R}^{d}} e^{Q_{c} f} d \mu \int_{\mathbb{R}^{d}} e^{-f} d \mu \leqslant 1 .
$$

Заметим, что из (2.2) и неравенства Йенсена следует

$$
\int_{\mathbb{R}^{d}} e^{Q_{c} f} d \mu \leqslant \exp \left(\int_{\mathbb{R}^{d}} f d \mu\right) .
$$

Легко проверить (см. [18], где был рассмотрен случай квадратичной функции стоимости), что если (2.2) выполнено, то (2.3) выполнено для любой $\mu$-интегрируемой $f$.

Хорошо известно (см. [11], [9]), что неравенство Талаграна

$$
\int_{\mathbb{R}^{d}} c(F) d \mu \leqslant \operatorname{Ent}_{\mu} g
$$

для выпуклой функции стоимости $c$ равносильно (2.3). 
Будем говорить, что вероятностная мера $\mu$ на $\mathbb{R}^{d}$ удовлетворяет логарифмическому с-неравенству Соболева, если для некоторого $\Lambda>0$ и любой локально липшицевой функции $f$

$$
\operatorname{Ent}_{\mu} f^{2} \leqslant \Lambda \int_{\mathbb{R}^{d}} c^{*}\left(\frac{\nabla f}{f}\right) f^{2} d \mu .
$$

Заметим, что для многих функций стоимости (2.4) влечет (2.3). В [19] было доказано, что произведение одномерных мер вида $e^{-|x|^{\alpha}} d x$ удовлетворяет $(2.4)$ для функции стоимости, равной $A \sum_{i=1}^{d}\left|x_{i}\right|^{\alpha}$ при больших значениях $x$ и квадратичной при малых $x$. Мы рассмотрим этот пример в следующем пункте.

Отметим, что существуют другие обобщения неравенства Соболева. Например, в [21] доказано, что для $\mu=e^{-|x|^{\alpha}} d x$ выполнено такое неравенство:

$$
\operatorname{Ent}_{\mu}|f|^{\beta} \leqslant c \int_{\mathbb{R}^{d}} \sum_{i}\left|\partial_{x_{i}} f\right|^{\beta} d \mu
$$

где $1 / \alpha+1 / \beta=1$.

В следующей лемме мы обобщаем результат из [22]. Доказательство близко к оригинальному, тем не менее, мы приведем его для удобства читателя.

ЛЕмма 1. Предположим, что вероятностная мера $\mu$ удовлетворяет следующему неравенству для любой локально липшищевой функиии $\varphi$ :

$$
\operatorname{Ent}_{\mu} e^{\varphi} \leqslant \frac{1}{2} \int_{X} H(\nabla \varphi) e^{\varphi} d \mu
$$

где $H: \mathbb{R}^{d} \rightarrow \mathbb{R}^{+}$обладает следующим свойством: для любого $x \in \mathbb{R}^{d}$ функция $\lambda \rightarrow H(\sqrt{\lambda} x)$ возрастающая и выпуклая на $[0, \infty)$ и $H(0)=0$. Тогда

$$
\int_{\mathbb{R}^{d}}\left(e^{\varphi-\int_{\mathbb{R}^{d}} \varphi d \mu}\right) d \mu \leqslant \int_{\mathbb{R}^{d}} e^{H(\nabla \varphi)} d \mu .
$$

В частности, если $t \rightarrow c^{*}(\sqrt{t} x)$ - выпуклая и неубывающая функиия на $[0, \infty)$ и $\mu$ удовлетворяет (2.4), то для любой локально липшищевой $\varphi$

$$
\int_{\mathbb{R}^{d}} \exp \left[\varphi-\int_{\mathbb{R}^{d}} \varphi d \mu\right] d \mu \leqslant \int_{\mathbb{R}^{d}} \exp \left(2 \Lambda c^{*}\left[\frac{\nabla \varphi}{2}\right]\right) d \mu .
$$

ДокАЗАТЕЛЬСТво. Положим

$$
g=H(\nabla \varphi)-\log \int_{\mathbb{R}^{d}} e^{H(\nabla \varphi)} d \mu .
$$

Заметим, что $\int_{\mathbb{R}^{d}} e^{g} d \mu=1$. По хорошо известному свойству энтропии

$$
\operatorname{Ent}_{\mu} f=\sup _{g}\left\{\int_{\mathbb{R}^{d}} f g d \mu: g \text { такова, что } \int_{\mathbb{R}^{d}} \exp (g) d \mu \leqslant 1\right\}
$$

получаем

$$
\int_{\mathbb{R}^{d}} e^{\varphi} g d \mu \leqslant \operatorname{Ent}_{\mu} e^{\varphi}
$$


Следовательно, (2.5) влечет

$$
2 \operatorname{Ent}_{\mu} e^{\varphi} \leqslant \int_{\mathbb{R}^{d}} e^{\varphi} H(\nabla \varphi) d \mu \leqslant\left(\int_{\mathbb{R}^{d}} e^{\varphi} d \mu\right) \log \left(\int_{\mathbb{R}^{d}} e^{H(\nabla \varphi)} d \mu\right)+\operatorname{Ent}_{\mu} e^{\varphi} .
$$

Таким образом,

$$
\operatorname{Ent}_{\mu} e^{\varphi} \leqslant\left(\int_{\mathbb{R}^{d}} e^{\varphi} d \mu\right) \log \left(\int_{\mathbb{R}^{d}} e^{H(\nabla \varphi)} d \mu\right) .
$$

Применим полученную формулу к $\lambda \varphi$ :

$$
\operatorname{Ent}_{\mu} e^{\lambda \varphi} \leqslant\left(\int_{\mathbb{R}^{d}} e^{\lambda \varphi} d \mu\right) \log \left(\int_{\mathbb{R}^{d}} e^{H(\lambda \nabla \varphi)} d \mu\right) .
$$

Положим

$$
K(\lambda)=\frac{1}{\lambda} \log \int_{\mathbb{R}^{d}} e^{\lambda \varphi} d \mu .
$$

Вычислим производную

$$
K^{\prime}(\lambda)=\frac{\operatorname{Ent}_{\mu} e^{\lambda \varphi}}{\lambda^{2} \int_{\mathbb{R}^{d}} e^{\lambda \varphi} d \mu} \leqslant \frac{\log \left(\int_{\mathbb{R}^{d}} e^{H(\lambda \nabla \varphi)} d \mu\right)}{\lambda^{2}} .
$$

Обозначим

$$
F(t)=\log \left(\int_{\mathbb{R}^{d}} e^{H(\sqrt{t} \nabla \varphi)} d \mu\right) .
$$

Очевидно, $F$ неотрицательна и не убывает. Покажем, что $F$ выпукла. Действительно, это следует из того факта, что сумма функций вида $\sum_{i=1}^{d} e^{W_{i}}$, где каждая $W_{i}$ выпукла, имеет вид $e^{W}$ для некоторой выпуклой функции $W$.

Принимая во внимание, что $F(0)=0$, получаем, что $F(t) / t$ не убывает. Следовательно,

$$
\lambda \rightarrow \frac{\log \left(\int_{\mathbb{R}^{d}} e^{H(\lambda \nabla \varphi)} d \mu\right)}{\lambda^{2}}
$$

не убывает. В итоге получаем

$$
\log \int_{\mathbb{R}^{d}} e^{\varphi} d \mu=K(1) \leqslant K(0)+\int_{0}^{1} K^{\prime}(\lambda) d \lambda \leqslant \int_{\mathbb{R}^{d}} \varphi d \mu+\log \left(\int_{\mathbb{R}^{d}} e^{H(\nabla \varphi)} d \mu\right),
$$

что и требовалось доказать. Неравенство (2.6) следует из доказанного неравенства и (2.4) путем замены $f^{2}=e^{g}$.

Теорема 2. Предположим, что с удовлетворяет условиям A1)-A4). Предположим также, что

1)

2) $t \rightarrow c^{*}(\sqrt{t} x)$ - выпуклая и возрастающая функиия на $[0, \infty)$ для всех $x$;

3) существует такое $N>0$, что

$$
\langle x, \nabla c(x)\rangle \leqslant N c(x)
$$


Тогда

$$
\int_{\mathbb{R}^{d}} e^{\omega c(F)} d \mu d \mu \leqslant\|g\|_{L^{p}(\mu)}
$$

где $\omega=\min (1 /(2 q), 1 /(4 \Lambda N))$.

ДокАЗАТЕльство. В силу неравенства $V(t x) \leqslant t V(x)$, выполненного для любой выпуклой функции $V$ с $V(0)=0$ и $0 \leqslant t \leqslant 1$, имеем

$$
c^{*}(\sqrt{t} x) \leqslant t c^{*}(x), \quad 0 \leqslant t \leqslant 1 .
$$

Тогда из неравенства $V(x) \leqslant\langle x, \nabla V(x)\rangle$ и условия 3) вытекает

$$
c^{*}(\tau x) \leqslant \tau^{2} c^{*}(x) \leqslant \tau^{2}\left\langle x, \nabla c^{*}(x)\right\rangle=\tau^{2}\left\langle\nabla c\left(\nabla c^{*}(x)\right), \nabla c^{*}(x)\right\rangle \leqslant N \tau^{2} c\left(\nabla c^{*}(x)\right) .
$$

Применим (2.6) к $\varepsilon \varphi$, где $\varphi$ - гладкая ограниченная функция:

$$
\int_{\mathbb{R}^{d}} \exp \left[\varepsilon \varphi-\int_{\mathbb{R}^{d}} \varepsilon \varphi d \mu\right] d \mu \leqslant \int_{\mathbb{R}^{d}} \exp \left(2 \Lambda c^{*}\left[\varepsilon \frac{\nabla \varphi}{2}\right]\right) d \mu \leqslant \int_{\mathbb{R}^{d}} \exp \left(\frac{\Lambda N \varepsilon^{2}}{2} c^{*}(\nabla \varphi)\right) d \mu .
$$

Из разложения в ряд Тейлора следует, что

$$
\int_{\mathbb{R}^{d}}\left(\varphi-\int_{\mathbb{R}^{d}} \varphi d \mu\right)^{2} d \mu \leqslant \frac{\Lambda N}{2} \int_{\mathbb{R}^{d}} c^{*}(\nabla \varphi) d \mu
$$

В силу (2.8)

$$
\int_{\mathbb{R}^{d}} c^{*}(\nabla \Phi) d \mu \leqslant N \int_{\mathbb{R}^{d}} c\left(\nabla c^{*}(\nabla \Phi)\right) d \mu=N \int_{\mathbb{R}^{d}} c(T(x)-x) d \mu .
$$

Так как (2.3) влечет неравенство Талаграна для функции стоимости $c$ и $\operatorname{Ent}_{\mu} g<\infty$, мы получаем $\int_{\mathbb{R}^{d}} c(T(x)-x) d \mu<\infty$ и $\Phi \in L^{2}(\mu)$. Выберем $\Phi$ таким образом, что $\int_{\mathbb{R}^{d}} \Phi d \mu=0$.

Пусть $\tau \geqslant 2 \omega>0$. По неравенству Юнга $x y \leqslant x \log x-x+e^{y}$, где $x \geqslant 0, y \in \mathbb{R}$, получаем

$$
\tau c(F) e^{\omega c(F)}=\tau\left(-\Phi-\Phi^{c}(T)\right) e^{\omega c(F)} \leqslant e^{-\tau \Phi}+e^{-\tau \Phi^{c}(T)}+2 \omega c(F) e^{\omega c(F)}-2 e^{\omega c(F)} .
$$

Следовательно, для любого $A \subset \mathbb{R}^{d}$

$$
(\tau-2 \omega) \int_{A} c(F) e^{\omega c(F)} d \mu+2 \int_{A} e^{\omega c(F)} d \mu \leqslant \int_{A} e^{-\tau \Phi} d \mu+\int_{A} e^{-\tau \Phi^{c}(T)} d \mu .
$$

Оценим правую часть следующим образом:

$$
\int_{\mathbb{R}^{d}} e^{-\tau \Phi^{c}(T)} d \mu=\int_{\mathbb{R}^{d}} e^{-\tau \Phi^{c}} g d \mu \leqslant\left(\int_{\mathbb{R}^{d}} g^{p} d \mu\right)^{1 / p}\left(\int_{\mathbb{R}^{d}} e^{-q \tau \Phi^{c}} d \mu\right)^{1 / q} .
$$

Предположим, что $\tau \leqslant 1 / q$. Из (2.3) следует, что

$$
\int_{\mathbb{R}^{d}} e^{-q \tau \Phi^{c}} d \mu \leqslant\left(\int_{\mathbb{R}^{d}} e^{-\Phi^{c}} d \mu\right)^{1 /(q \tau)} \leqslant e^{1 /(\tau q) \int_{\mathbb{R}^{d}} \Phi d \mu}=1,
$$


следовательно,

$$
\int_{\mathbb{R}^{d}} e^{-\tau \Phi^{c}(T)} d \mu=\int_{\mathbb{R}^{d}} e^{-\tau \Phi^{c}} g d \mu \leqslant\left(\int_{\mathbb{R}^{d}} g^{p} d \mu\right)^{\frac{1}{p}} .
$$

Далее положим $A:=A_{n}=\{x:-\Phi(x) \leqslant n\}$. Очевидно, $\nabla[\max (\Phi,-n)]=\nabla \Phi \chi_{A_{n}}$ $\mu$-п.в. Тогда по $(2.8)$ и $(2.6)$ имеем

$$
\begin{aligned}
\int_{A_{n}} e^{-\tau \Phi} d \mu & \leqslant \int_{\mathbb{R}^{d}} \exp (-\tau \max (\Phi,-n)) d \mu \\
& \leqslant \int_{\mathbb{R}^{d}} \exp \left(2 \Lambda c^{*}\left(\frac{\tau}{2} \nabla \Phi \chi_{A_{n}}\right)\right) d \mu \exp \left(-\tau \int_{\mathbb{R}^{d}} \max (\Phi,-n) d x\right) \\
& \leqslant \int_{\mathbb{R}^{d}} \exp \left(\frac{\Lambda N \tau^{2}}{2} c\left[\nabla c^{*}\left(\nabla \Phi \chi_{A_{n}}\right)\right]\right) d \mu \exp \left(-\tau \int_{\mathbb{R}^{d}} \max (\Phi,-n) d x\right) \\
& =\left(\int_{A_{n}} e^{\frac{\Lambda N \tau^{2}}{2} c(F)} d \mu+1-\mu\left(A_{n}\right)\right) \exp \left(-\tau \int_{\mathbb{R}^{d}} \max (\Phi,-n) d x\right) .
\end{aligned}
$$

Заметим, что для $x \in A_{n}$ выполнено

$$
c(T(x)-x)=-\Phi(x)-\Phi^{c}(T(x)) \leqslant n-\Phi^{c}(T(x)) .
$$

Положим $\tau=\min (1 / q, 1 /(2 \Lambda N))$. Таким образом, $\Lambda N \tau^{2} / 2 \leqslant \tau / 4 \leqslant 1 /(4 q)$ и

$$
\chi_{A_{n}} e^{\Lambda N \tau^{2} c(F) / 2} \leqslant e^{\tau n / 4} e^{-\tau \Phi^{c}(T) / 4} .
$$

Согласно полученной выше оценке функция $\chi_{A_{n}} e^{\Lambda N \tau^{2} c(F) / 2}$ интегрируема и

$$
\begin{aligned}
(\tau-2 \omega) & \int_{A_{n}} c(F) e^{\omega c(F)} d \mu+2 \int_{A_{n}} e^{\omega c(F)} d \mu \leqslant\left(\int_{\mathbb{R}^{d}} g^{p} d \mu\right)^{1 / p} \\
& +\left(\int_{A_{n}} e^{\Lambda N \tau^{2} c(F) / 2} d \mu+1-\mu\left(A_{n}\right)\right) \exp \left(\tau \int_{\mathbb{R}^{d}} \max (\Phi,-n) d x\right) .
\end{aligned}
$$

Положив $\omega:=\tau / 2$, получаем $\Lambda N \tau^{2} / 2 \leqslant \tau / 2 \leqslant \omega$ и

$$
\begin{aligned}
& 2 \int_{A_{n}} e^{\omega c(F)} d \mu \leqslant\left(\int_{\mathbb{R}^{d}} g^{p} d \mu\right)^{1 / p} \\
& \quad+\left(\int_{A_{n}} e^{\omega c(F)} d \mu+1-\mu\left(A_{n}\right)\right) \exp \left(\tau \int_{\mathbb{R}^{d}} \max (\Phi,-n) d x\right) .
\end{aligned}
$$

Принимая во внимание, что $\lim _{n \rightarrow \infty} \int_{\mathbb{R}^{d}} \max (\Phi,-n) d x=0$, получаем утверждение теоремы.

Теорема 3. Предположим, что с удовлетворяет условиям А1)-A4) и влетворяет неравенству инфимум-свертки (2.3) и неравенству Пуанкаре (1.3). Предположим, что

1) $\int_{\mathbb{R}^{d}} g|\log g|^{p} d \mu<\infty$ для некоторого $p \geqslant 1$;

2) существуют такие $p^{\prime} \geqslant 1 u N_{p^{\prime}}>0, M_{p^{\prime}}>0$, ито $p^{\prime} \geqslant p u|\nabla c|^{2 p^{\prime}} \leqslant$ $N_{p^{\prime}} c^{p}+M_{p^{\prime}}$. 
Тогда существуют такие $A, B>0$, зависящие от $p, C, N_{p^{\prime}}, M_{p^{\prime}}$, что

$$
\int_{\mathbb{R}^{d}} c^{p}(F) d \mu \leqslant A+B \int_{\mathbb{R}^{d}} g|\log g|^{p} d \mu
$$

ДокАЗАтЕЛьство. Идея доказательства та же самая, что и в теореме 2. Мы дадим ниже формальные оценки, из которых следует результат. Более подробное доказательство может быть проведено так же, как и в теореме 2.

Из соотношения $c(x-T(x))=-\Phi-\Phi^{c}(T)$ следует, что

$$
\int_{\mathbb{R}^{d}} c^{p}(x-T(x)) d \mu=-\int_{\mathbb{R}^{d}} c^{p-1}(x-T(x))\left(\Phi+\Phi^{c}(T)\right) d \mu .
$$

Согласно 2) $|\nabla \Phi|^{2 p^{\prime}} \leqslant M_{p^{\prime}}+N_{p^{\prime}} c^{p}(F)$. Следовательно, $|\nabla \Phi|^{2 p^{\prime} / p} \in L^{1}(\mu)$, и по неравенству Пуанкаре $\Phi \in L^{2}(\mu)$. Выберем $\Phi$ таким образом, что

$$
\int_{\mathbb{R}^{d}} \Phi d \mu=\log \int_{\mathbb{R}^{d}} c^{p-1}(x-T(x)) d \mu
$$

Тогда по неравенству Юнга

$$
\begin{gathered}
-\int_{\mathbb{R}^{d}} \Phi^{c}(T(x)) c^{p-1}(x-T(x)) d \mu=-\int_{\mathbb{R}^{d}} \Phi^{c}(x) c^{p-1}\left(x-T^{-1}(x)\right) g d \mu \\
\leqslant \int_{\mathbb{R}^{d}} e^{-\Phi^{c}(x)} d \mu+\int_{\mathbb{R}^{d}} c^{p-1}\left(x-T^{-1}(x)\right) g \log \left[c^{p-1}\left(x-T^{-1}(x)\right) g\right] d \mu \\
\quad-\int_{\mathbb{R}^{d}} c^{p-1}\left(x-T^{-1}(x)\right) g d \mu .
\end{gathered}
$$

Используя (2.3), мы получаем

$$
\int_{\mathbb{R}^{d}} e^{-\Phi^{c}} d \mu \leqslant e^{\int_{\mathbb{R}^{d}} \Phi d \mu}=\int_{\mathbb{R}^{d}} c^{p-1}(x-T(x)) d \mu .
$$

Следовательно,

$$
\begin{aligned}
-\int_{\mathbb{R}^{d}} \Phi^{c}(T(x)) c^{p-1}(x-T(x)) d \mu \leqslant & \int_{\mathbb{R}^{d}} c^{p-1}(x-T(x)) \log \left[c^{p-1}(x-T(x)) g(T)\right] d \mu \\
= & \int_{\mathbb{R}^{d}} c^{p-1}(x-T(x)) \log \left[c^{p-1}(x-T(x))\right] d \mu \\
& +\int_{\mathbb{R}^{d}} c^{p-1}(x-T(x)) \log g(T) d \mu .
\end{aligned}
$$

По неравенству Гёльдера и формуле замены переменных

$$
\int_{\mathbb{R}^{d}} c^{p-1}(x-T(x)) \log g(T(x)) d \mu \leqslant\left[\int_{\mathbb{R}^{d}} c^{p}(x-T(x)) d \mu\right]^{1-1 / p}\left[\int_{\mathbb{R}^{d}}|\log g|^{p} g d \mu\right]^{1 / p} .
$$


Кроме того,

$$
\begin{gathered}
-\int_{\mathbb{R}^{d}} \Phi(x) c^{p-1}(x-T(x)) d \mu=-\int_{\mathbb{R}^{d}} c^{p-1}(x-T(x)) d \mu \log \left(\int_{\mathbb{R}^{d}} c^{p-1}(x-T(x)) d \mu\right) \\
-\int_{\mathbb{R}^{d}}\left(\Phi(x)-\int_{\mathbb{R}^{d}} \Phi d x\right) c^{p-1}(x-T(x)) d \mu \\
\leqslant-\int_{\mathbb{R}^{d}} c^{p-1}(x-T(x)) d \mu \log \left(\int_{\mathbb{R}^{d}} c^{p-1}(x-T(x)) d \mu\right) \\
+\left[\int_{\mathbb{R}^{d}} c^{p}(x-T(x)) d \mu\right]^{1-1 / p}\left[\int_{\mathbb{R}^{d}}\left|\Phi(x)-\int_{\mathbb{R}^{d}} \Phi d x\right|^{p} d \mu\right]^{1 / p} .
\end{gathered}
$$

Заметим, что любая мера, удовлетворяющая неравенству Пуанкаре, удовлетворяет также следующему неравенству для $p^{\prime} \geqslant 1$ и подходящей константы $C_{2 p^{\prime}}$ :

$$
\int_{\mathbb{R}^{d}}\left|\varphi-\int_{\mathbb{R}^{d}} \varphi d \mu\right|^{2 p^{\prime}} d \mu \leqslant C_{2 p^{\prime}} \int_{\mathbb{R}^{d}}|\nabla \varphi|^{2 p^{\prime}} d \mu
$$

(см., например, [21]). По неравенству Гёльдера

$$
\begin{aligned}
\int_{\mathbb{R}^{d}} \mid \Phi- & \left.\int_{\mathbb{R}^{d}} \Phi d x\right|^{p} d \mu \leqslant\left(\int_{\mathbb{R}^{d}}\left|\Phi-\int_{\mathbb{R}^{d}} \Phi d x\right|^{2 p^{\prime}} d \mu\right)^{p /\left(2 p^{\prime}\right)} \\
& \leqslant\left(\int_{\mathbb{R}^{d}} C_{2 p^{\prime}}|\nabla \Phi|^{2 p^{\prime}} d \mu\right)^{p /\left(2 p^{\prime}\right)}=C_{2 p^{\prime}}^{p / 2 p^{\prime}}\left(\int_{\mathbb{R}^{d}}|\nabla c(F)|^{2 p^{\prime}} d \mu\right)^{p /\left(2 p^{\prime}\right)} \\
& \leqslant C_{2 p^{\prime}}^{p / 2 p^{\prime}}\left(\int_{\mathbb{R}^{d}} N_{p^{\prime}} c^{p}(F) d \mu+M_{p^{\prime}}\right)^{p /\left(2 p^{\prime}\right)} .
\end{aligned}
$$

В конечном итоге получаем, что при подходящем выборе констант $A^{\prime}>0, B^{\prime}>0$

$$
\begin{aligned}
-\int_{\mathbb{R}^{d}} \Phi c^{p-1}(F) d \mu \leqslant A^{\prime} & {\left[\int_{\mathbb{R}^{d}} c^{p}(F) d \mu\right]^{1-1 / p+1 /\left(2 p^{\prime}\right)} } \\
& +B^{\prime}-\int_{\mathbb{R}^{d}} c^{p-1}(F) d \mu \log \left(\int_{\mathbb{R}^{d}} c^{p-1}(F) d \mu\right)
\end{aligned}
$$

и

$$
\begin{aligned}
-\int_{\mathbb{R}^{d}} \Phi^{c}(T) c^{p-1}(F) d \mu \leqslant \int_{\mathbb{R}^{d}} & c^{p-1}(F) \log \left[c^{p-1}(F)\right] d \mu \\
+ & {\left[\int_{\mathbb{R}^{d}} c^{p}(F) d \mu\right]^{1-1 / p}\left[\int_{\mathbb{R}^{d}}|\log g|^{p} g d \mu\right]^{1 / p} . }
\end{aligned}
$$

Таким образом, $\int_{\mathbb{R}^{d}} c^{p}(F) d \mu$ не превосходит суммы правых частей этих неравенств

$$
\begin{aligned}
\int_{\mathbb{R}^{d}} c^{p}(F) d x \leqslant A^{\prime} & {\left[\int_{\mathbb{R}^{d}} c^{p}(F) d \mu\right]^{1-1 / p+1 /\left(2 p^{\prime}\right)}+B^{\prime}+\operatorname{Ent}_{\mu} c^{p-1}(F) } \\
+ & {\left[\int_{\mathbb{R}^{d}} c^{p}(F) d \mu\right]^{1-1 / p}\left[\int_{\mathbb{R}^{d}}|\log g|^{p} g d \mu\right]^{1 / p} . }
\end{aligned}
$$

Утверждение теоремы следует из полученной оценки. 
ЗАмечАниЕ 1. 1) В качестве примера функций стоимости, удовлетворяющих условиям теоремы 2 и теоремы 3 , можно взять $c(x)=|x|^{p}$, где $1<p \leqslant 2$.

2) Пусть $c=|x|^{2}$. Тогда теорема 1 следует из теоремы 2 и леммы 1.

3) Сделаем несколько замечаний об условиях теорем 2 и 3 . Не очень ясно, какова связь между неравенствами (2.3) и (2.6). Второе из них, как мы видели, следует из (2.4). Во многих ситуациях то же верно для (2.3). Условие 2) теоремы 3 следует из условий 2), 3) теоремы 2. Действительно, из неравенства $c^{*}(t x) \leqslant t^{2} c^{*}(x)$ получаем, что $c^{*}(x) \geqslant|x|^{2} \inf _{|x|=1} c^{*}(x)=a|x|^{2}$ при $|x| \geqslant 1$. Тогда по (2.8), если $|\nabla c| \geqslant 1$, то $a^{2 p}|\nabla c|^{2 p} \leqslant\left(c^{*}(\nabla c)\right)^{p} \leqslant N^{p} c^{p}(x)$. Следовательно, $|\nabla c|^{2 p} \leqslant 1+\left(N^{p} / a^{p}\right) c^{p}$.

3. Примеры. Пусть $\alpha \geqslant 1$. Зададим следующую вероятностную меру на $\mathbb{R}^{d}$ :

$$
\mu_{\alpha}=\frac{1}{Z_{\alpha}^{d}} \prod_{i=1}^{d} e^{-\left|x_{i}\right|^{\alpha}} d x_{i}
$$

где $Z_{\alpha}=\int_{\mathbb{R}} e^{-|x|^{\alpha}} d x$.

Спектральные свойства этих мер впервые изучались в [23]. В частности, в [23] было показано, что для $\mu_{\alpha}$ выполнена следующая серия неравенств, которые можно рассматривать как интерполяцию между неравенством Пуанкаре и логарифмическим неравенством Соболева:

$$
\int_{\mathbb{R}^{d}} f^{2} d \mu_{\alpha}-\left(\int_{\mathbb{R}^{d}}|f|^{p} d \mu_{\alpha}\right)^{2 / p} \leqslant C(2-p)^{2(1-1 / \alpha)} \int_{\mathbb{R}^{d}}|\nabla f|^{2} d \mu_{\alpha} .
$$

Дальнейшее развитие этой тематики см. в [24], [25]. В нашей работе мы будем использовать результаты, полученные недавно в [19].

В [19] было показано, что $\mu_{\alpha}$ удовлетворяет транспортному неравенству для функций стоимости следующего вида:

$$
L_{A, \alpha}^{d}(x)=\sum_{i=1}^{d} L_{A, \alpha}\left(x_{i}\right),
$$

где $2 \geqslant \alpha>1, A>0,1 / \alpha+1 / \beta=1$ и

$$
L_{A, \alpha}(x)= \begin{cases}\frac{x^{2}}{2}, & \text { если }|x| \leqslant A, \\ A^{2-\alpha} \frac{|x|^{\alpha}}{\alpha}+A^{2} \frac{\alpha-2}{2 \alpha}, & \text { если }|x| \geqslant A .\end{cases}
$$

Можно показать, что

$$
\left(L_{A, \alpha}^{d}\right)^{*}=H_{A, \alpha}^{d}, \quad\left(H_{A, \alpha}^{d}\right)^{*}=L_{A, \alpha}^{d},
$$

где

$$
H_{A, \alpha}^{d}(x)=\sum_{i=1}^{d} H_{A, \alpha}\left(x_{i}\right), \quad H_{A, \alpha}(x)= \begin{cases}\frac{x^{2}}{2}, & \text { если }|x| \leqslant A, \\ A^{2-\beta} \frac{|x|^{\beta}}{\beta}+A^{2} \frac{\beta-2}{2 \beta}, & \text { если }|x| \geqslant A .\end{cases}
$$

В дальнейшем мы опустим индекс $d$ и будем писать $L_{A, \alpha}, H_{A, \alpha}$.

Сформулируем основной результат из [19]. 
Теорема 4. Следующие неравенства выполнены для $\mu_{\alpha}$.

1) (Логарифмическое $c$-неравенство Соболева) Существует такая константа $C_{\alpha}>0$, что для любого $f \in C_{0}^{\infty}\left(\mathbb{R}^{d}\right)$

$$
\operatorname{Ent}_{\mu_{\alpha}} f^{2} \leqslant C_{\alpha} \int_{\mathbb{R}^{d}} H_{A, \alpha}\left(\frac{\nabla f}{f}\right) f^{2} d \mu_{\alpha} .
$$

2) (Транспортное неравенство) Для любой вероятностной меры $g \cdot \mu_{\alpha}$

$$
T_{\frac{A C_{\alpha}, \alpha}{2}}\left(\mu_{\alpha}, g \cdot \mu_{\alpha}\right) \leqslant \frac{C_{\alpha}}{4} \operatorname{Ent}_{\mu_{\alpha}} g
$$

где

$$
T_{L_{\frac{A C_{\alpha}}{2}, \alpha}}\left(\mu_{\alpha}, g \cdot \mu_{\alpha}\right)=\inf _{\pi}\left\{\int_{\mathbb{R}^{2 d}} L_{\frac{A C_{\alpha}}{2}, \alpha}(x-y) d \pi(x, y)\right\},
$$

а инбимум берется по множеству вероятностных мер $\pi$ на $\mathbb{R}^{d} \times \mathbb{R}^{d}$, причем проекции $\pi$ на первый и второй сомножитель совпадают соответственно с $g \cdot \mu_{\alpha} u$ $\mu_{\alpha}$.

3) (Неравенство инфимум-свертки) Для любой ограниченной измеримой $\varphi$

$$
\int_{\mathbb{R}^{d}} e^{Q \varphi} d \mu_{\alpha} \leqslant \exp \left(\int_{\mathbb{R}^{d}} \varphi d \mu_{\alpha}\right)
$$

¿əe

$$
Q \varphi=\inf _{y}\left\{\varphi(y)+\frac{4}{C_{\alpha}} L_{\frac{T C_{\alpha}}{2}, \alpha}(x-y)\right\} .
$$

В действительности 2) и 3 ) следуют из 1). Если $\alpha=2$, мы получаем классическое логарифмическое неравенство Соболева и транспортное неравенство для гауссовской меры. Заметим, что этот результат также доказан для случая $\alpha=1$ и функции стоимости

$$
L_{A, 1}^{d}(x)=\sum_{i=1}^{d} L_{A, 1}\left(x_{i}\right)
$$

где

$$
L_{A, 1}(x)=\left\{\begin{array}{ll}
\frac{x^{2}}{2}, & \text { если }|x| \leqslant A, \\
A|x|-\frac{A^{2}}{2}, & \text { если }|x| \geqslant A,
\end{array} \quad H_{A, 1}(x)=L_{A, 1}^{*}(x)= \begin{cases}\frac{x^{2}}{2}, & \text { если }|x| \leqslant A, \\
\infty, & \text { если }|x| \geqslant A .\end{cases}\right.
$$

ПРЕДЛОЖЕНИЕ 1. Пусть $g \cdot \mu_{\alpha}$ - вероятностная мера и $T(x)=x+F(x)-$ onтимальное отображение, минимизирующее функиию стоимости $c=L_{\frac{A C_{\alpha}}{2}, \alpha} u$ отображающее $\mu_{\alpha}$ в $g \cdot \mu_{\alpha}$.

1) Если $g \in L^{p}\left(\mu_{\alpha}\right)$ для некоторого $p>1$, то

$$
\int_{\mathbb{R}^{d}} e^{\varepsilon c(F)} d \mu_{\alpha} \leqslant\|g\|_{L^{p}\left(\mu_{\alpha}\right)}
$$

для некоторого $\varepsilon=\varepsilon(\alpha, p)>0$.

2) Если $g|\log g|^{p} \in L^{1}\left(\mu_{\alpha}\right)$ для некоторого $p>1$, то

$$
\int_{\mathbb{R}^{d}} c^{p}(F) d \mu \leqslant A(\alpha, p)+B(\alpha, p) \int_{\mathbb{R}^{d}} g|\log g|^{p} d \mu_{\alpha} .
$$


ДокАЗАтельство. Для доказательства 1) применим теорему 2. Докажем, что все условия на $c$ выполнены. Действительно, A1)-A4) и условие 3) теоремы 2 нетрудно проверить. Заметим, что для $d=1$

$$
H_{A, \alpha}(x)=\max \left\{f_{1}(x), f_{2}(x)\right\}, \quad \text { где } \quad f_{1}(x)=\frac{x^{2}}{2}, \quad f_{2}(x)=A^{2-\beta} \frac{|x|^{\beta}}{\beta}+A^{2} \frac{\beta-2}{\beta} .
$$

Так как функции $t \rightarrow f_{1}(\sqrt{t} x), \quad t \rightarrow f_{2}(\sqrt{t} x)$ выпуклы, мы получаем, что $t \rightarrow$ $c^{*}(\sqrt{t} x)$ - выпуклая и возрастающая функция. Условие 3$)$ очевидным образом выполнено.

Хорошо известно, что $\mu_{\alpha}$ удовлетворяет неравенству Пуанкаре (см. [19]). Покажем, что неравенство (2.6) вытекает из (3.1). По теореме 3 для любой гладкой функции $f$

$$
\int_{\mathbb{R}^{d}} \exp \left(f-\int_{\mathbb{R}^{d}} f d \mu_{\alpha}\right) d \mu_{\alpha} \leqslant \int_{\mathbb{R}^{d}} \exp \left(2 C_{\alpha} H_{A, \alpha}\left(\frac{\nabla f}{2}\right)\right) d \mu_{\alpha} .
$$

Заметим, что для $A \leqslant A^{\prime}$ и подходящего $M\left(A, A^{\prime}\right) \geqslant 1$ получаем $H_{A, \alpha} \leqslant H_{A^{\prime}, \alpha} \leqslant$ $M\left(A, A^{\prime}\right) H_{A, \alpha}$. Следовательно, (2.6) выполнено также для функции $H_{\frac{A C_{\alpha}, \alpha}{2}}=c^{*}$ и подходящего $\Lambda>0$. Неравенство (2.3) для функции стоимости $4 /\left(C_{\alpha}\right) L_{\frac{T C_{\alpha}}{2}, \alpha}$ следует из теоремы (3.2). Следовательно, оно выполнено и для $L_{\frac{T C_{\alpha}}{2}, \alpha}$ с точностью до константы $4 /\left(C_{\alpha}\right)$. Читатель легко проверит, что теорема 2 выполнена также и в этом случае. Пункт 1) доказан.

Пункт 2) легко следует из теоремы 3 , условия которой выполнены согласно замечанию 1.

Автор выражает благодарность рецензенту за важные замечания.

\section{СПИСОК ЦИТИРОВАННОЙ ЛИТЕРАТУРЫ}

[1] Y. Brenier, "Polar factorization and monotone rearrangement of vector valued functions", Comm. Pure Appl. Math., 44 (1991), 375-417.

[2] R. J. McCann, "Existence and uniqueness of monotone measure-preserving maps", Duke Math. J., 80 (1995), 309-323.

[3] L. Ambrosio, N. Gigli, G. Savaré, Gradient Flows in Metric Spaces and in the Wasserstein Spaces of Probability Measures, Lectures in Math., ETH, Zürich, 2005.

[4] S. T. Rachev, L. Rüschendorf, Mass Transportation Problems, I, II, Springer, New York, 1998.

[5] C. Villani, Topics in Optimal Transportation, Amer. Math. Soc., Providence, RI, 2003.

[6] L. Gross, "Logarithmic Sobolev inequalities", Amer. J. Math., 97:4 (1975), 1061-1083.

[7] В. И. Богачев, Основы теории меры, 1, 2, РХД, Москва-Ижевск, 2003.

[8] F. Y. Wang, "Logarithmic Sobolev inequalities on non-compact Riemannian manifolds", Probab. Theory Related Fields, 109 (1997), 417-424.

[9] M. Ledoux, The Concentration of Measure Phenomenon, Amer. Math. Soc., Providence, RI, 2001.

[10] C. Ané, S. Blachère, D. Chafai, P. Fougère, I. Gentil, F. Malrieu, C. Roberto, G. Scheffer, Sur les inégalités de Sobolev logarithmiques, Panorama et Synthèses, 10, Soc. Math. France, Paris, 2002.

[11] S. G. Bobkov, I. Gentil, M. Ledoux, "Hypercontractivity of Hamilton-Jacobi equations", J. Math. Pures Appl., 80:7 (2001), 669-696. 
[12] M. Talagrand, "Transportation cost for Gaussian and other product measures", Geom. Funct. Anal., 6 (1996), 587-600.

[13] F. Otto, C. Villani, "Generalization of an inequality by Talagrand, and links with the logarithmic Sobolev inequality", J. Funct. Anal., 173 (2000), 361-400.

[14] Y. Bolley, C. Villani, "Weighted Csiszár-Kullback-Pinsker inequalities and applications to transportation cost inequalities", Ann. Faculté des Sciences de Toulouse, 14 (2005), 331-352.

[15] H. Djellout, A. Guillin, L. Wu, "Transportation cost-information inequalities and applications to random dynamical systems and diffusions", Ann. Probab., 32:3B (2004), 27022732 .

[16] P. Cattiaux, A. Guillin, "Talagrand's like quadratic transportation cost inequalities", Preprint, 2004.

[17] X. Fernique, "Extension du théorème de Cameron-Martin aux translations aléatoires. II. Intégrabilité des densités", High Dimensional Probability, III (Sandjberg, 2002), Progr. Probab., 55, Birkhäuser, Basel, 2003, 95-102.

[18] В. И. Богачев, А. В. Колесников, "Интегрируемость абсолютно непрерывных преобразований мер и применения к оптимальной транспортировке масс", Теория вероятн. и ее примен., 50:3 (2005), 3-25.

[19] I. Gentil, A. Guillin, L. Miclo, "Modified logarithmic Sobolev inequalities and transportation inequalities", Probab. Theory Related Fields, 133:3 (2005), 409-436.

[20] W. Gangbo, R. J. McCann, "The geometry of optimal transportation", Acta Math., 177 (1996), 113-161.

[21] S. G. Bobkov, B. Zegarlinski, "Entropy bounds and isoperimetry", Mem. Amer. Math. Soc., 176:829 (2005).

[22] S. G. Bobkov, F. Götze, "Exponential integrability and transportation cost related to logarithmic Sobolev inequality", J. Funct. Anal., 163 (1999), 1-28.

[23] R. Latała, K. Oleskiewicz, "Between Sobolev and Poincaré", Geometric Aspects of Functional Analysis, Lecture Notes in Math., 1745, Springer, Berlin, 2000, 147-168.

[24] F. Barthe, P. Cattiaux, C. Roberto, "Interpolated inequalities between exponential and Gaussian, Orlicz hypercontractivity and isoperimetry", Revista Mat. Iberoamericana (to appear); E-print math.PR/0407219, 2004.

[25] F. Y. Wang, "Probability distance inequalities on Riemannian manifolds and path spaces", J. Funct. Anal., 206 (2004), 167-190.

А. В. Колесников

Московский государственный университет

им. М. В. Ломоносова

E-mail: sascha77@mail.ru
Поступило

10.10.2005

Исправленный вариант

03.06.2006 